\title{
The application of amplitude-preserved processing and migration for carbonate reservoir prediction in the Tarim Basin, China
}

\author{
Sam Zandong Sun ${ }^{1 *}$, Yang Haijun ${ }^{2}$, Zhang Yuanyin ${ }^{1}$, Han Jianfa ${ }^{2}$, Wang \\ Dan $^{1}$, Sun Wenbo ${ }^{1}$ and Jiang Shan ${ }^{1}$ \\ ${ }^{1}$ Laboratory for Integration of Geology \& Geophysics, China University of Petroleum, Beijing 102249, China \\ ${ }^{2}$ Research Institute of Exploration \& Development, PetroChina Tarim Oilfield Company, Korla 841000, China \\ (C) China University of Petroleum (Beijing) and Springer-Verlag Berlin Heidelberg 2011
}

\begin{abstract}
Conventional seismic exploration method based on post-stack data usually fails to identify the distribution of fractured and caved carbonate reservoirs in the Tarim Basin, so the rich pre-stack information should be applied to the prediction of carbonate reservoirs. Amplitude-preserved seismic data processing is the foundation. In this paper, according to the feature of desert seismic data (including weak reflection, fast attenuation of high frequency components, strong coherent noises, low $\mathrm{S} / \mathrm{N}$ and resolution), a set of amplitude-preserved processing techniques is applied and a reasonable processing flow is formed to obtain the high quality data. After implementing a set of pre-stack amplitude-preserved processing, we test and define the kernel parameters of amplitude-preserved Kirchhoff PSTM (pre-stack time migration) and subsequent gathers processing, in order to obtain the amplitude-preserved gathers used to the isotropic pre-stack inversion for the identification of caved reservoirs. The AVO characteristics of obtained gathers fit well with the synthetic gathers from logging data, and it proves that the processing above is amplitudepreserved. The azimuthal processing techniques, including azimuth division and binning enlargement, are implemented for amplitude-preserved azimuthal gathers with the uniform fold. They can be used in the anisotropic inversion to detect effective fractures. The processing techniques and flows are applied to the field seismic data, and are proved available for providing the amplitude-preserved gathers for carbonate reservoir prediction in the Tarim Basin.
\end{abstract}

Key words: Amplitude-preserved processing, amplitude-preserved pre-stack time migration, azimuth, carbonate reservoir, Tarim Basin

\section{Introduction}

With further development of exploration and the increase in successful wells in carbonate reservoirs, complicated carbonate reservoirs which are deeply buried in the Tarim Basin are gradually becoming important exploration goals. The conventional method of dissolved cave identification based on bead-like reflections from seismic profiles fails to determine the filling features of the storage space, and it usually neglects other heterogeneous reservoirs. Therefore, more rich pre-stack seismic information should be employed to do AVO analysis and elastic inversion. So a technique to obtain high quality and amplitude-preserved pre-stack data is the key to the pre-stack data analysis. Ordinary methods like coherence cube could not predict effective fractures (open or filled by fluid), and small cracks are usually neglected. Moreover, although the shear wave data could recognize

\footnotetext{
* Corresponding author. email: samzdsun@yahoo.com
}

Received March 18, 2011 fractures effectively, its considerable expense makes the method difficult to implement widely. Considering the fact that the field data acquisition is azimuthally limited, a method of obtaining the amplitude-preserved processing flow which is used for fracture detection would be very important. The aim of early processing does not serve for the pre-stack analysis, the amplitude-preserved capability is unsatisfactory, and the processing does not take anisotropy into consideration. Additionally, most of the techniques could not solve the problem of multiples elimination, relative amplitude preservation, fractures and caves imaging, so that they could not satisfy the need in reservoir prediction and oil $\&$ gas detection (Tao et al, 2010).

Considering the surface conditions and raw data characteristics in the Tarim Basin, the processing difficulties in deep layers are analyzed at first in this paper, and then a set of amplitude-preserved data processing workflow for reservoir characterization is summarized and explained, based on former research and practical application (Guo, 2009; Wang et al, 2008). Under the guidance of this workflow, the 
resolution and amplitude-preservation are improved, which lays the foundation for reservoir prediction.

\section{Processing challenges of desert data and amplitude-preserved processing workflow}

\subsection{Processing challenges of desert data}

For data processing in the Tarim Basin, many problems usually exist and can be listed as the following aspects: (1) Although the conventional shooting method is replaced by exploding below the water table, seismic data still have poor quality resulted from the complex desert surface conditions. The seismic data are featured as weak reflection, fast attenuation of high frequency components, strong coherent noises, and low S/N and resolution. (2) Processing and interpretation are heavily influenced by multiples produced by the low velocity layer in the desert surface. (3) The reflection of secondary carbonate reservoirs is always accompanied with complicated scattering and diffraction which need to be eliminated by a reasonable migration technique ( $\mathrm{Li}$ et al, 2007). (4) Due to the effects of tectonic movement and weathering erosion, spatial structure characteristics in the subsurface are complicated and variable, and it is hard to identify their effective seismic reflection energy. So, special amplitude-preserved processing techniques should be employed. (5) Since the development of carbonate reservoirs is affected by many factors such as sedimentation, tectonic movement and diagenesis, the secondary fractures and dissolved caves with variable sizes and complicated shapes are well developed (Zhao et al, 2007; Wu et al, 2005; Zhou et al, 2008). Therefore, anisotropic information should be considered.

\subsection{Amplitude-preserved processing workflow}

Precisely imaging structure, predicting lithological reservoir and detecting hydrocarbon, and monitoring residual oil-gas distribution during the development of oil-gas fields are the three basic targets for data processing. Amplitudepreserved processing is the foundation of the techniques of isotropic AVO inversion, anisotropic inversion, reservoir feature analysis and the detection of oil and gas.

For the amplitude-preserved processing which is used for the prediction of fractured and caved carbonate reservoirs, the most important factor is to eliminate the diffraction waves which appear around caves and fractures, image the reflection energy, and make sure the seismic data have correct amplitude. Additionally, we should also do well in the static correction, amplitude-preserved noise attenuation, amplitude compensation, surface consistent deconvolution and precise velocity analysis and residual static correction.

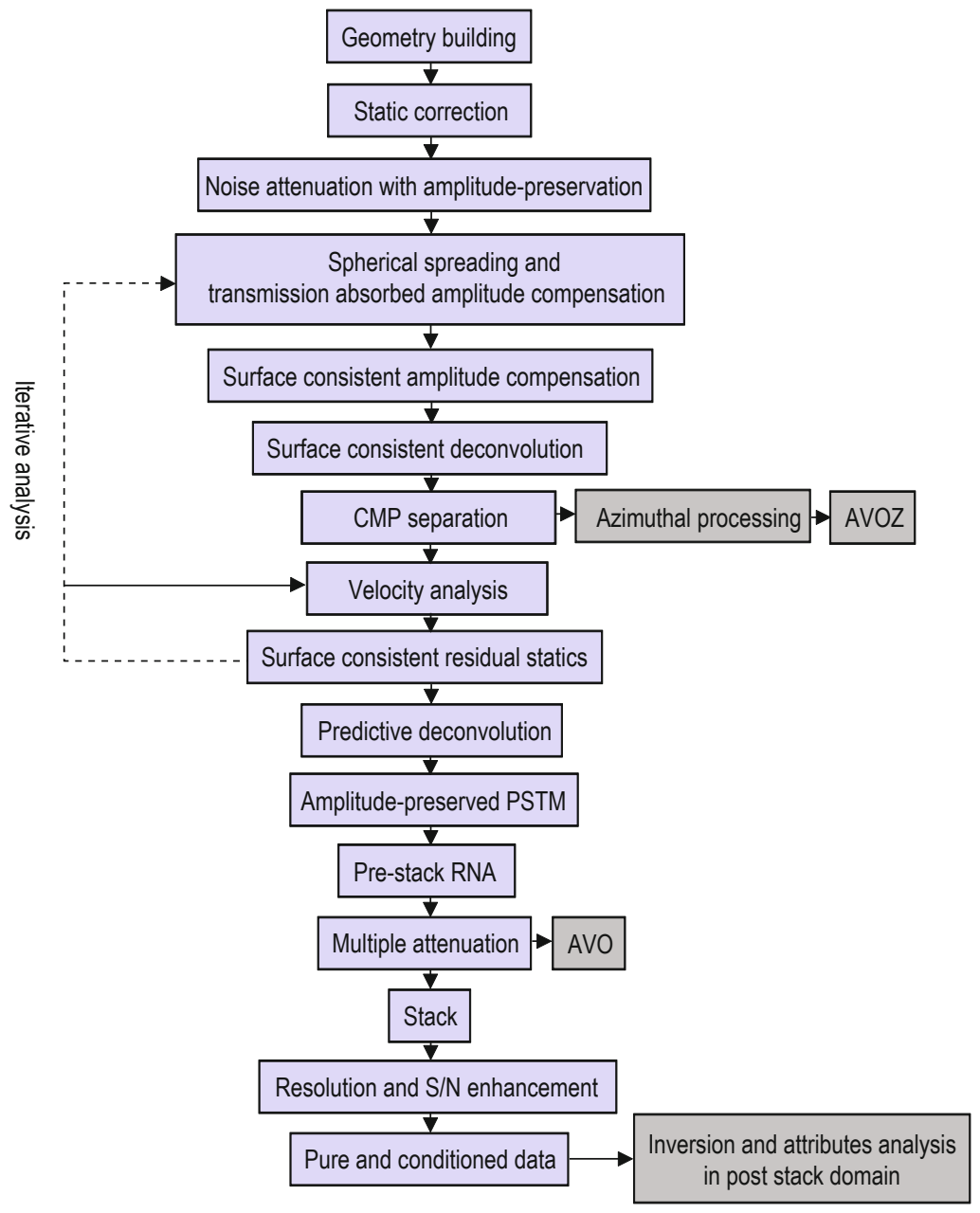

Fig. 1 The workflow of amplitude-preserved processing (RNA: random noise attenuation) 
The high quality seismic data which have preserved the information of heterogeneous features could be used in the pre-stack AVO inversion to predict caved reservoirs. For a fractured carbonate reservoir with strong anisotropy, considering the limited azimuth data acquisition and the need in AVOZ inversion, we performed azimuthal processing before migration, and then amplitude-preserved migration and the subsequent processing with data of each azimuth were finished. Finally we obtained the azimuth data which can be used in the anisotropy analysis. Focusing on carbonate reservoir prediction, a series of processing techniques aiming at obtaining the amplitude-preserved seismic data is introduced in this paper, and the flow is shown in Fig. 1. The results from isotropic and anisotropic inversion using the data from this flow both reflect the distribution characteristics of caved and fractured carbonate reservoirs which illustrates the effectiveness of this strategy.

\section{Key amplitude-preserved techniques}

\subsection{Multiples suppression}

Multiples and random noise are prominent in the seismic data from the Tarim Basin (Fig. 2). In CRP (common reflection point) gathers, multiples are more obvious than those in CMP (common middle point) gathers. In order to perform pre-stack analysis, these multiples should be effectively removed, and Radon transform method is often used. For random noise, TDNFK (3D noise attenuation in F-K domain) noise elimination method is preferable. The results of multiples suppression are shown in Fig. 2. Fig. 2(a) is the CRP gather after migration, and Fig. 2(b) is CRP gather after eliminating the multiples, and Fig. 2(c) is the multiples removed. Fig. 2(d), (e), and (f) are the corresponding velocity spectrums of Fig. 2(a), (b), and (c). It can be clearly observed that the multiples have been effectively suppressed.

\subsection{The sequence of TDNFK and migration}

The pre-stack migration is quite sensitive to noise, which can be enlarged after migration. Therefore, pre-stack noise elimination is the foundation for the following data processing (Zhang, 2006). The real seismic data in the Tarim Basin are of poor quality. They show strong surface waves, linear waves, multi-refraction waves and random noises. Several noise suppression techniques are integrated to enhance the $\mathrm{S} / \mathrm{N}$
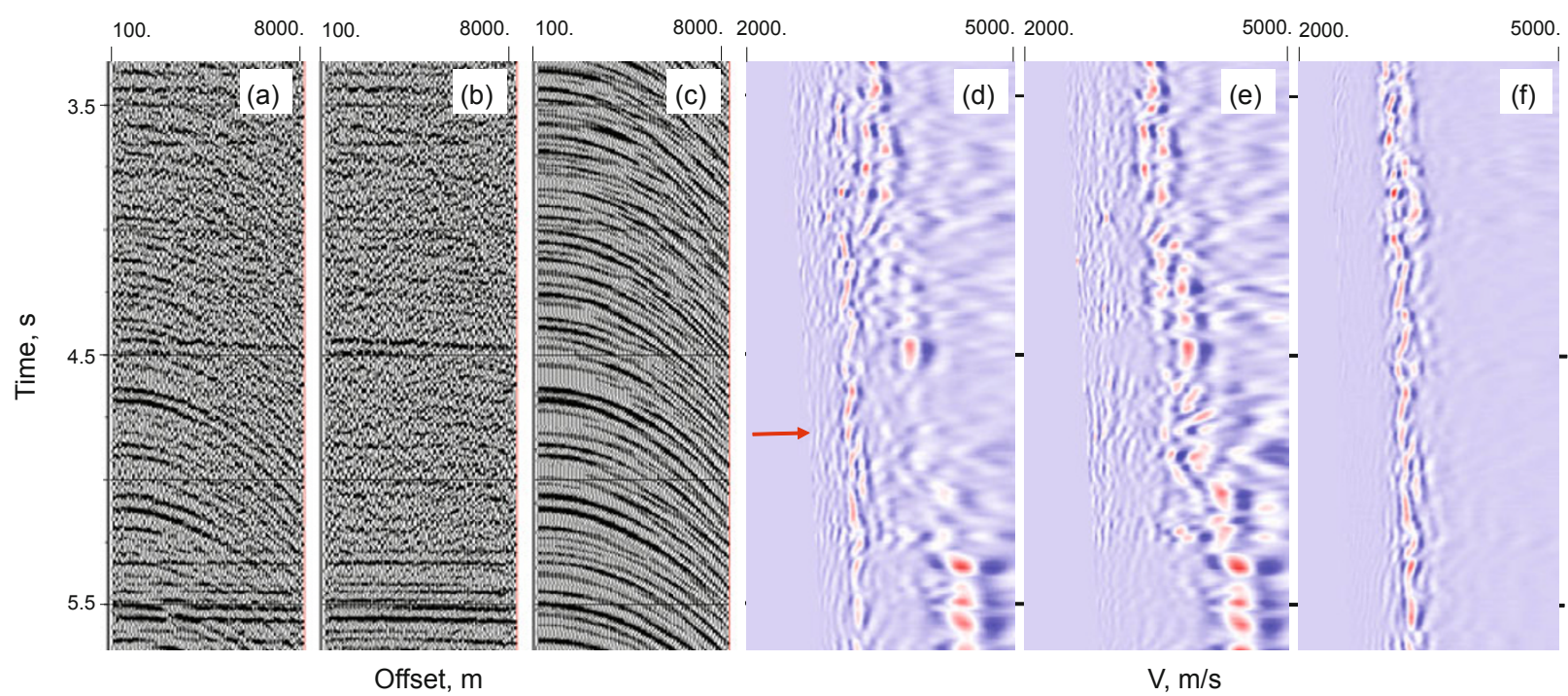

Fig. 2 Comparison of CRP before and after multiples suppression

ratio, and at the same time, to maintain the effective reflection signal. The linear waves are removed according to the velocity difference, and the anomalous amplitude is suppressed using a median filter. The TDNFK method is applied for surface wave suppression in $f-K_{x} K_{y}$ domain. When this method is conducted before and after migration respectively, different imaging results can be obtained, as shown in Fig. 3. It is better for TDNFK to be performed after migration. In the amplitude-preserved data processing, noise is subtracted from the original data, which is beneficial for amplitude preservation.

\subsection{Amplitude-preserved Kirchhoff pre-stack time migration}

Amplitude-preserved seismic data are required to correctly perform attribute analysis and AVO/AVA inversion (Sun, 2002). In 1993, the Kirchhoff amplitude-preserved migration method was put forward by Schleicher (Schleicher et al, 1993). Furthermore, in 1999 Graham A. Winbow (Graham et al, 1999) provided an explicit 3D amplitude-preserved weight function formula for pre-stack time migration and utilized true amplitude weight function estimation to compensate amplitude. In this paper, the pre-stack Kirchhoff amplitudepreserved migration is applied in the field data of this area, and we mainly analyzed and tested the main parameters of this amplitude-preserved migration method in order to obtain high quality seismic data for pre-stack inversion.

\subsubsection{The selection of key parameters}

(1) Regroup offset

Firstly, the maximum and the minimum offset and the 

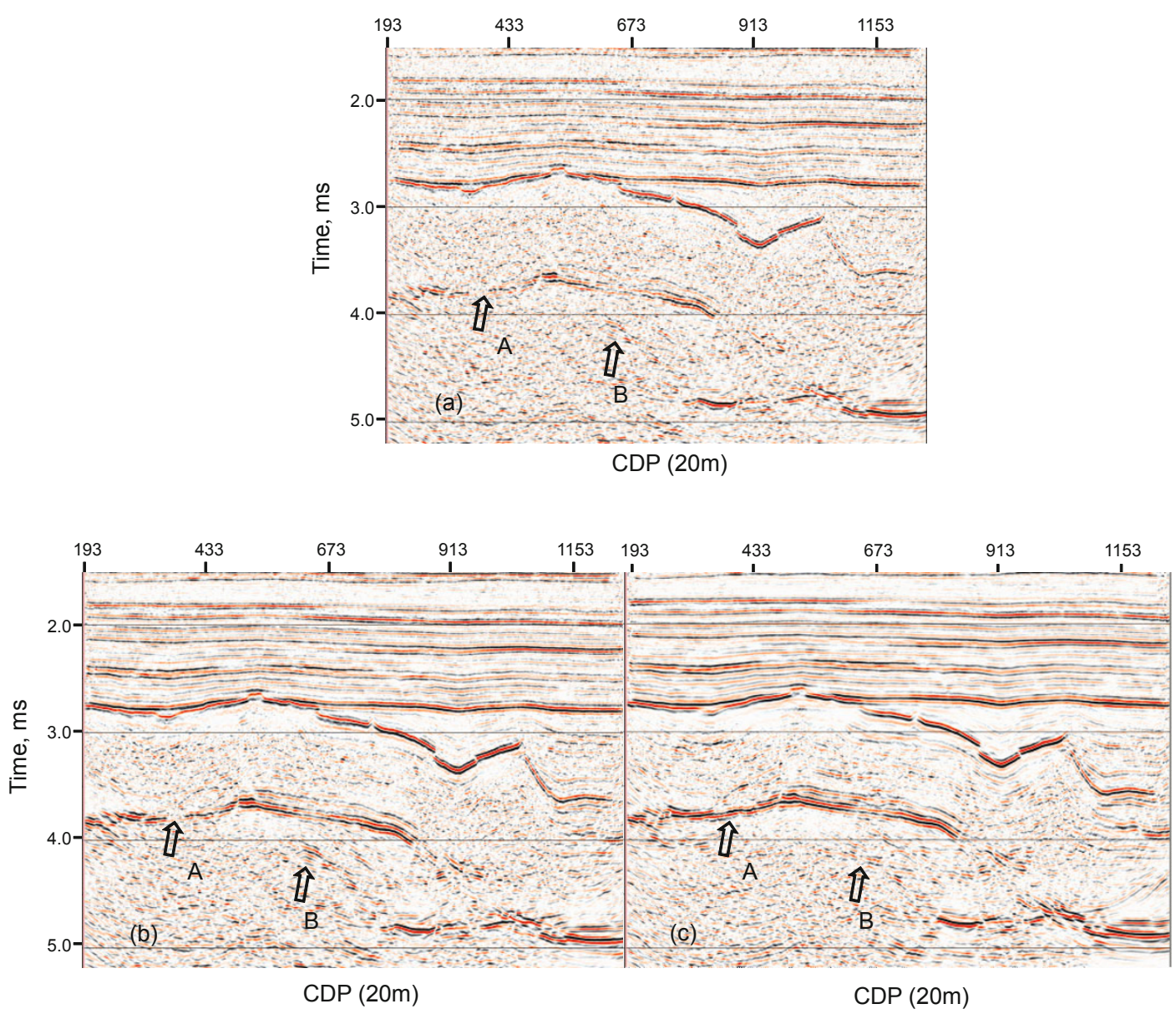

Fig. 3 The influence of noise elimination sequence on imaging

(a) Pre-stack time migration section (b) Noise elimination after migration (c) Noise elimination before migration

offset increment are achieved by investigating and analyzing the offset distribution of CMP gathers. Particularly, missing traces in the same offset should be less than $30 \%$, and the offset distribution of every CMP gather should be uniform and as complete as possible.

(2) Migration aperture

The migration aperture refers to the seismic data distribution for the migration. In general, the migration aperture is controlled by three factors: the maximum dip and depth of reflection interface, input data length and ray path aperture. It was said that a small aperture may only ensure structure imaging effects for small dip data and characteristics of the high $\mathrm{S} / \mathrm{N}$, but not for steep angle data which at the same time would have structural distortion and give rise to the "flattened" phenomenon. A large aperture can ensure structural imaging for steep dip, but the continuity of the reflection section would become worse, and the $\mathrm{S} / \mathrm{N}$ and resolution would fall (Chen et al, 2002).

(3) The angle of migration

The maximum migration angle refers to the maximum angle to be preserved during the migration process. On one hand, it limits the scope of the migration operator extensions in the space. On the other hand, it has an influence on the migration arithmetic as well as computing times. The larger the migration angle, the more the computing times. Through the corresponding parameter testing carried out for different structural regions in the Tarim Basin, the dip of layer is usually small. Therefore, in order to ensure the recognition of local small structures, the protected dip value is chosen to be 45 degrees.

(4) Ray path

The calculation of reflection travel time can be approximately simplified as a straight line with a lower calculation precision. The ray bending method calculates travel time according to the interval velocity model which is based on the Snell's Law ray tracing method with high accuracy for complex and complicated areas. According to the geological characteristics of the Tarim Basin and computing ability, the bending travel time calculation method should be selected.

(5) Anti-alias filtering

The frequency of the migration section and imaging quality can be directly influenced by anti-frequency parameters. If frequency is too high, high-frequency noise will be introduced and false frequency phenomenon will be caused, and if frequency is too low, high frequency components will be lost. Aliasing always appears at the shallow section, so an anti-alias filter is needed. We limit the highest frequency to the scope of the effective wave and adopt anti-alias filtering. 


\subsubsection{Analysis of migration and processing results}

In the course of amplitude-preserved PSTM, signals can be imaged appropriately through choosing a proper directional factor, spherical divergence factor and phase adjustment factor. For imaging of carbonate reservoirs in the Tarim Basin, amplitude-preserved PSTM is the most important. The different CRP gathers by different Kirchhoff migration algorithms are selected and shown in Fig. 4. The amplitudes at $5.0 \mathrm{~ms}$ of different CRP gathers are extracted, and are shown in Fig. 5. We can notice that the amplitudes marked by red and green lines are much more similar and reasonable, and are advantageous for inversion.

In order to test the amplitude preservation after data processing and migration, the CRP gather after noise elimination and migration is compared with the theoretical synthetics, which is shown in Fig. 6. The reasonability of amplitude-preserved processing is affirmed by the similar AVO characteristics between synthetics and CRP gathers, especially in the target area marked by the red frame. Prestack inversion identifying carbonate reservoirs using the prestack gathers from this processing flow shows reasonable results.
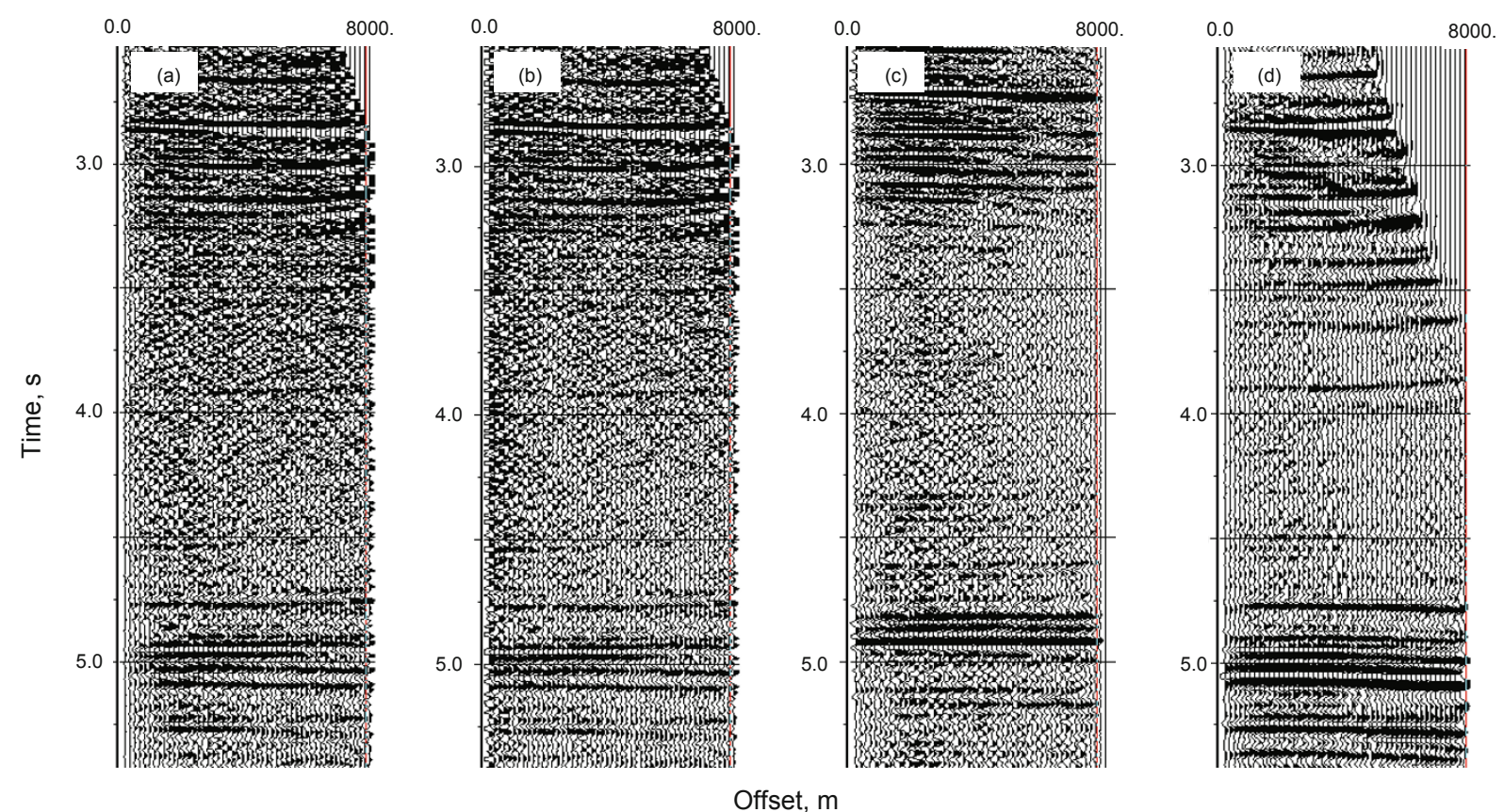

Fig. 4 CRP gathers by different migration algorithms

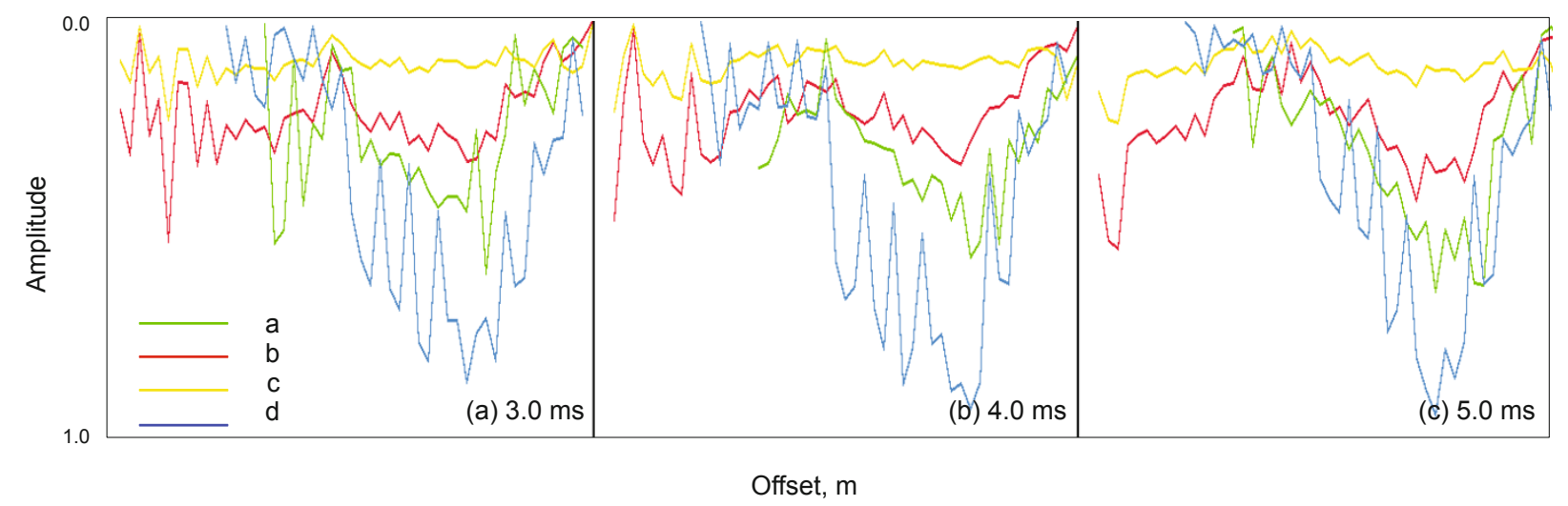

Fig. 5 Amplitudes extracted from different CRP gathers in Fig. 4

\section{Azimuth amplitude-preserved Kirchhoff PSTM}

The P-wave is sensitive to anisotropic media, and it is featured by frequency loss, and changes of amplitude, phase, velocity and fault imaging. These features are in favor of extracting the information from anisotropic reservoir (Williams and Jenner, 2002). Although the scale of terranes or 


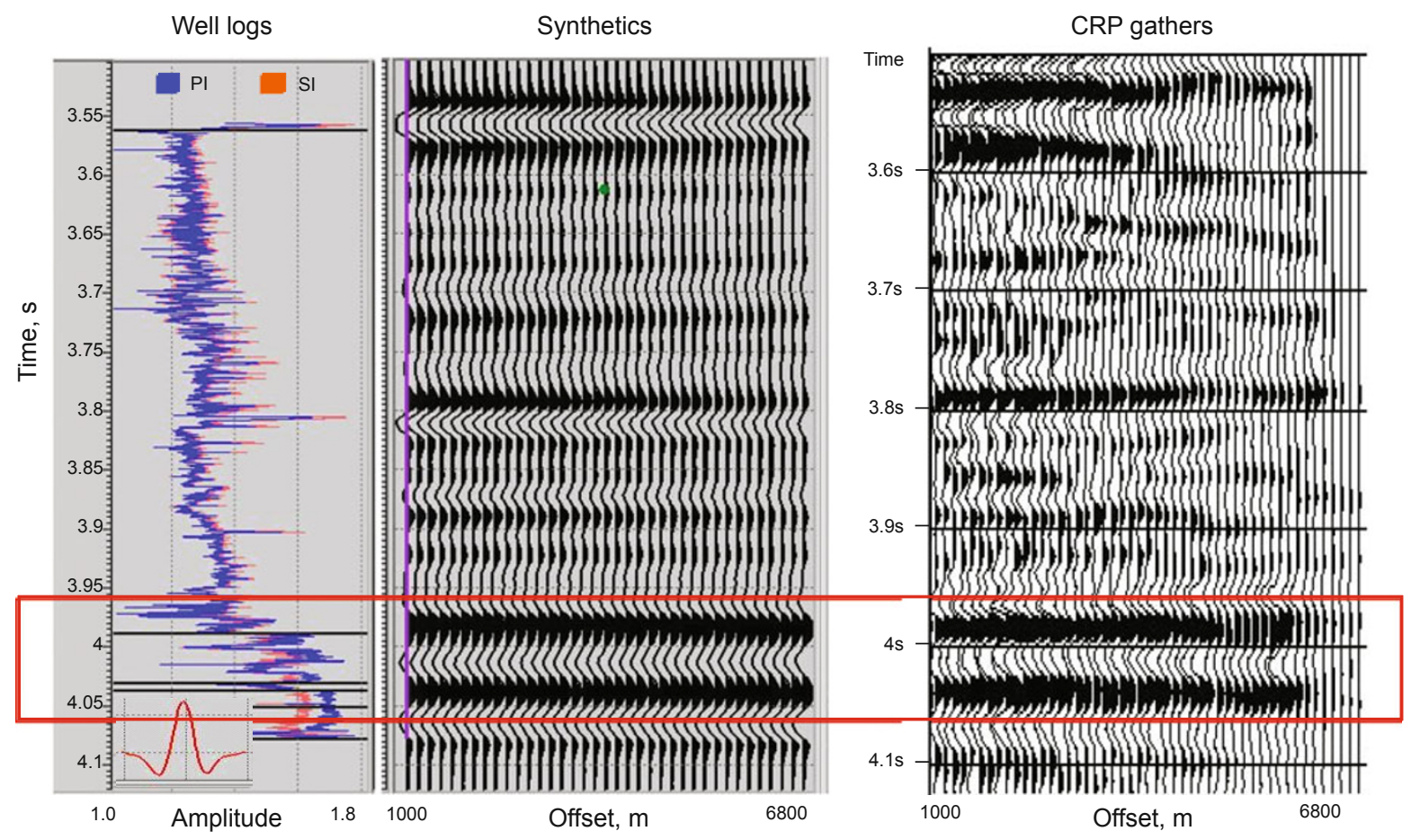

Fig. 6 Comparison between synthetics and PSTM CRP gathers

fissures is less than seismic wavelength, the macro anisotropic effects still can be observed (Jenner, 2002). Actually, there is a definite relationship between the amplitude changes versus azimuth and anisotropy. Specifically, if fractures in a certain azimuth exist in the subsurface, the AVO characteristics of CRP gathers in the azimuths parallel with and normal to fractures are obviously different. The distribution and development densities of fractures can be reflected by the size and distribution of that difference (Zhang et al, 2004). Based on this, combined with the data in the Tarim Basin, the azimuth range is chosen through analyzing the azimuth, offset and fold times of CMP gathers at first, and then the bins are enlarged to improve S/N and fold times. Finally, amplitudepreserved PSTM and other processing are applied to the azimuthal gathers.

\subsection{Azimuth and offset analysis, and azimuth division}

Considering the high cost, seismic data acquisition in the Tarim Basin is generally not applied to the whole azimuth. Therefore, the distribution of azimuth-offset is not uniform and usually lacks far offset components in some azimuths (Fig. 7). There are two basic messages: (1) The mean aspect ratio in this area is about 0.45 (Fig. 7(a)). (2) Through analyzing the fold distribution versus azimuth (Fig. 7(b)), it is exhibited as a shape of two peaks. About $66 \%$ data are distributed in the range between positive $30^{\circ}$ and negative $30^{\circ}$ azimuth (including corresponding opposite direction $150^{\circ}-210^{\circ}$, the east is regarded as $0^{\circ}$, increasing anti-clockwise). Finally, to meet the demand of anisotropic inversion, the azimuth angles from $-30^{\circ}$ to $30^{\circ}$ are divided into four groups of commonazimuth gathers for processing, and each group differentiates $15^{\circ}$ (Fig. 8(a)). The offsets and azimuths of bins can not be guaranteed to uniform in the acquisition in this area, which will lead to AVO error. To avoid this and improve $\mathrm{S} / \mathrm{N}$, the bin size is enlarged. The data bin in the Tarim Basin can usually be enlarged from $20 \mathrm{~m} \times 20 \mathrm{~m}$ to $40 \mathrm{~m} \times 40 \mathrm{~m}$ (Fig. $8(\mathrm{~b})$ ).

\subsection{Azimuth velocity analysis and residual static correction}

Since the velocity and static measurement are different in different azimuth data, velocity analysis and residual static correction should be performed respectively in different azimuth data to protect the anisotropic characteristics before migration. Then amplitude-preserved PSTM method discussed above is applied to all these azimuth data respectively.

\subsection{Common azimuth amplitude-preserved PSTM}

Based on the azimuth data divided in Fig. 8, the corresponding four common azimuth gathers are conducted migration. The four CRP gathers after PSTM and noise attenuation are shown in Fig. 9, which locate at the red dashed lines in the corresponding stack sections in Fig. 10. Obviously, the azimuthal processing is successful since there are more distinct AVO characteristics in CRP gathers, better wave features and more continuous events in the stack section. In addition, the anisotropic characteristics of different common azimuth data are outstanding, which are suitable for anisotropic inversion. The pre-stack anisotropic inversion using azimuthal data from this processing flow shows good results in effective fracture detection. 

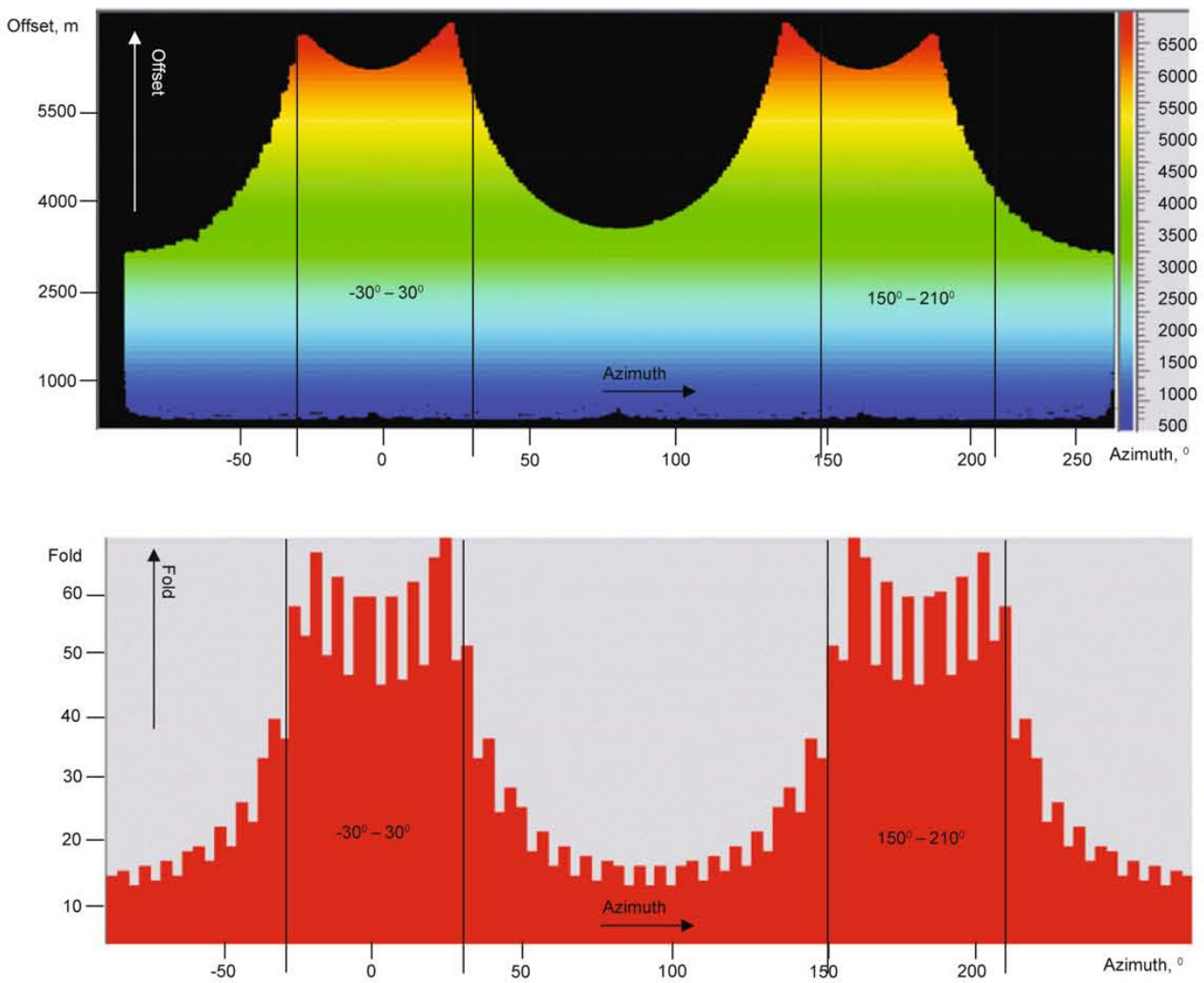

Fig. 7 (a) Offset vs. azimuth distribution analysis

(b) Offset vs. fold distribution analysis
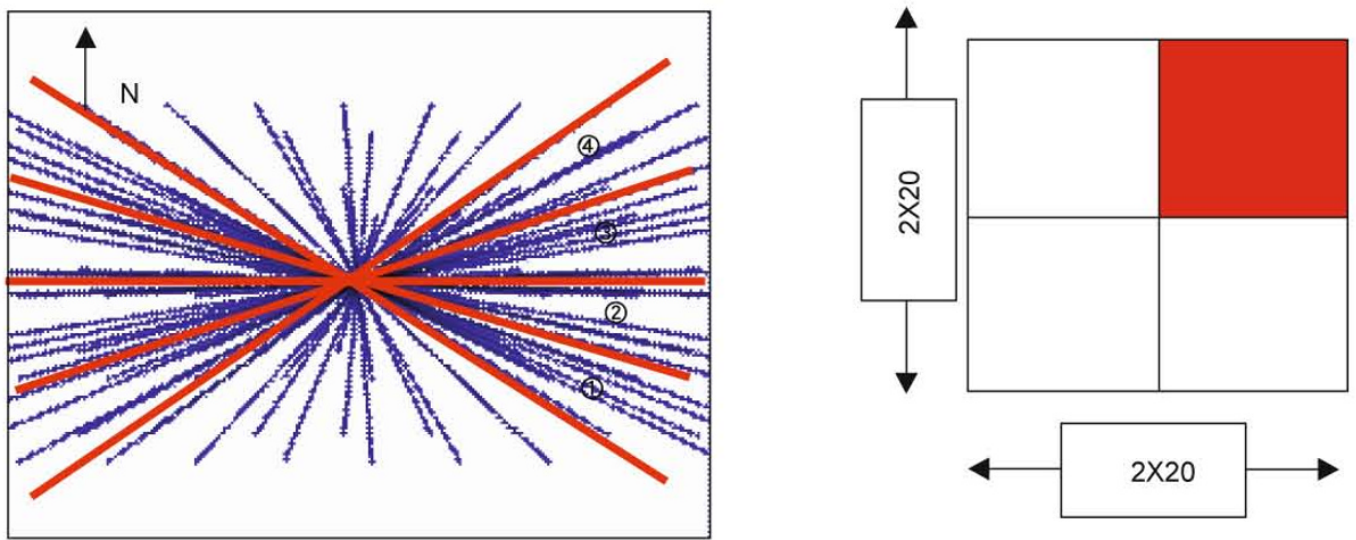

Fig. 8 (a) Azimuth distribution rules

(b) Common reflection bin expanding method

\section{Conclusions}

The exploration study of Tarim Basin is a systematic seismic investigation involving acquisition, processing and interpretation. It needs consistent working thought. To begin with, seismic data acquisition in desert area should try to avoid sand dunes to guarantee the fold times, and excite under the water table to guarantee deep reflection energy. Besides, small bins and wide azimuth should be adopted when designing the survey system. Finally, data processing should be applied for the target of interpretation, and the effective information should be extracted economically and efficiently. From our experience, the following should be noted:

(1) The whole set of amplitude-preserved processing flow include static correction, combined noise attenuation, spherical divergence and surface consistent amplitude compensation, sequence deconvolution, iteration of precise velocity analysis and residual static correction, amplitude- 

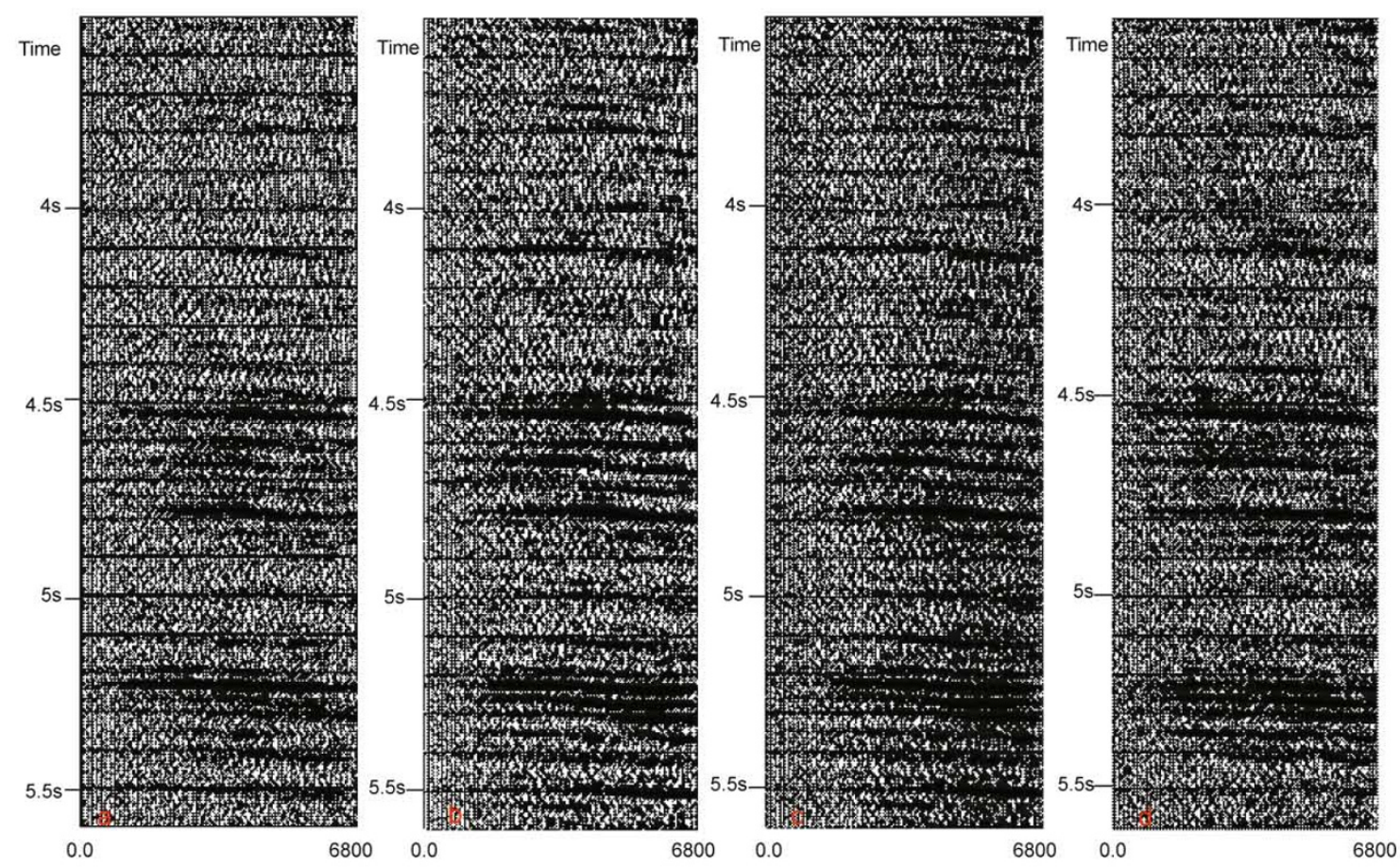

Offset, $\mathrm{m}$

Fig. 9 Four sets of different common azimuth gathers after PSTM and noise attenuation (a) $-30^{\circ}-15^{\circ}$; (b) $-15^{\circ}-0^{\circ}$; (c) $0^{\circ}-15^{\circ}$; (d) $15^{\circ}-30^{\circ}$

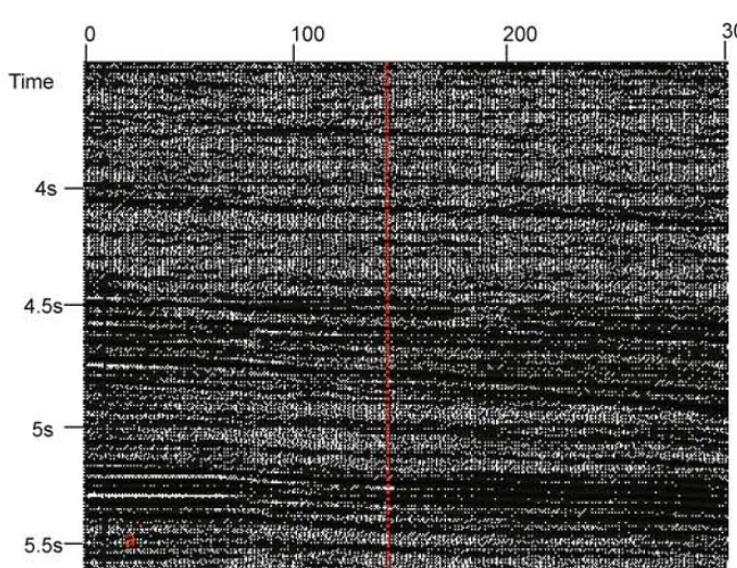

CDP

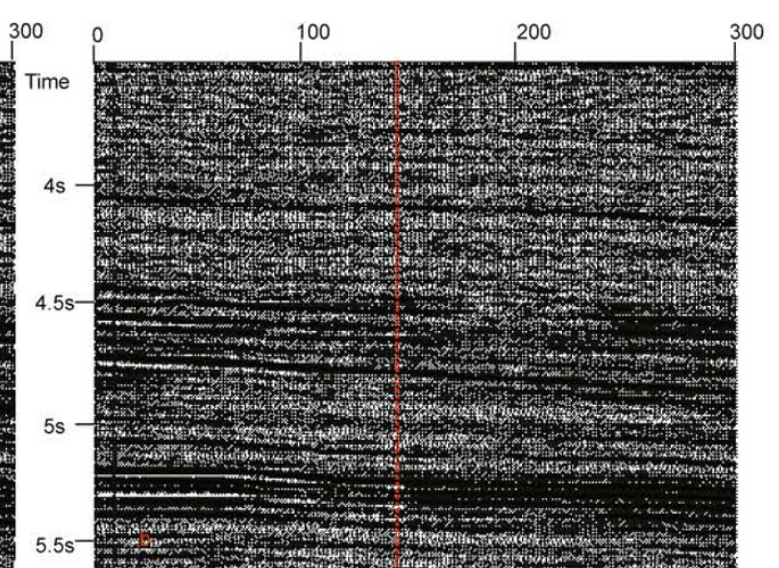

CDP

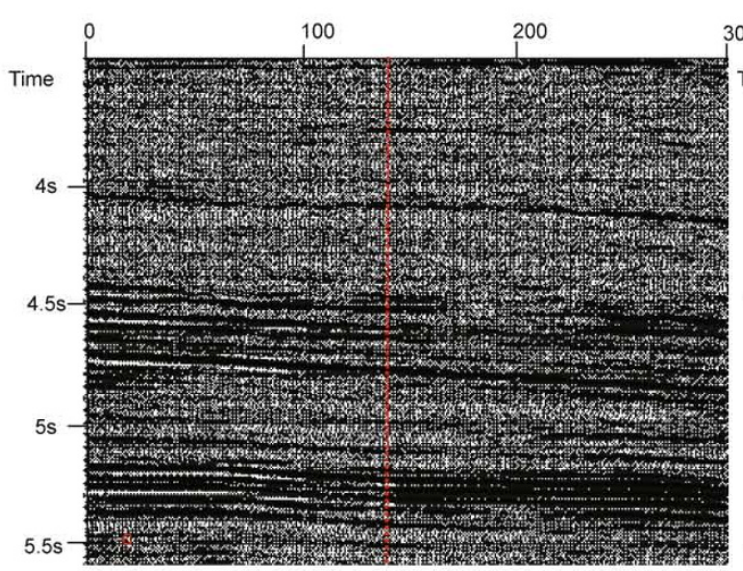

CDP
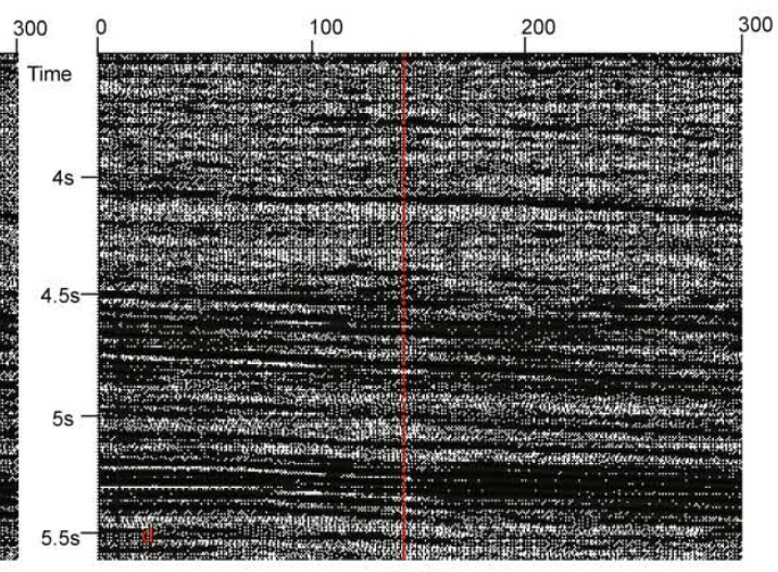

CDP

Fig. 10 Four different common azimuth sections after PSTM and noise attenuation (a) $-30^{\circ}-15^{\circ}$; (b) $-15^{\circ}-0^{\circ}$; (c) $0^{\circ}-15^{\circ}$; (d) $15^{\circ}-30^{\circ}$ 
preserved PSTM and the following noise attenuation should be applied consistently. Attention should be paid to the preservation of effective reflection amplitude and quality control in each step.

(2) The amplitude-preserved pre-stack time migration technique is the key to the accurate imaging of carbonate reservoirs in the Tarim Basin. This affects the later pre-stack inversion and reservoir prediction. In order to obtain an image of carbonate reservoirs in the Tarim Basin, the migration aperture was tested and finally defined as $6,000 \mathrm{~m}$, and the effective imaged dip angle reached 45 degrees.

(3) For fracture prediction through anisotropic inversion based on P-wave data, it is an economic and effective method to realize processing common azimuth gathers, which is a new direction of fracture prediction. To avoid fold nonuniform and improve $\mathrm{S} / \mathrm{N}$, the bin size, used to azimuth migration, needs to be enlarged when seismic data are divided by different azimuths.

\section{Acknowledgements}

This research is financially supported by National Basic Research Program of China (No.2011CB201100). The data were provided by Exploration and Development Research Institution of Tarim Oil Field Company. The authors would like to thank Wang Di and Yang Pei for much work of proofreading.

\section{References}

Chen Z D, Liu Z K and Li C B. 3D pre-stack depth migration velocity analysis and automatic Monte Carlo velocity picking in depth. Chinese Journal of Geophysics. 2002. 45(2): 246-254 (in Chinese)

Graham A W, William A and Schneider J. Weights for 3-D controlled amplitude prestack time migration. SEG Expanded Abstracts 18. 1999. 1110-1113

Guo S X. Discussion on preserved amplitude processing of seismic data.
Petroleum Geophysics. 2009. 7(1): 1-3

Jenner E. Fractured reservoir characterization using P-wave AVOA analysis of 3D OBC data. The Leading Edge. 2002. 21(8): 777-781

Li G H, Gao H L, Zhang Z Q, et al. Reservoir prediction for the east of Lun-Gu area. In: Thesis Collections of the Integration of Exploration and Development for Lun-Gu Oilfield in the Tarim Basin. Beijing: Petroleum Industry Press. 2007 (in Chinese)

Schleicher J, Tygel M and Hubral P. 3-D true-amplitude finite-offset migration. Geophysics. 1993. 58(8): 1112-1126

Sun J G. Kirchhoff-type true-amplitude migration and demigration. Progress in Exploration Geophysics. 2002. 25(6): 1-5 (in Chinese)

Tao Y G, Wang X W, Lü L, et al. Seismic data pre-stack processing techniques for carbonate reservoirs in the central Tarim Basin. Oil Geophysical Prospecting. 2010. 45(2): 230-236 (in Chinese)

Wang X W, Lü L, Liu W F, et al. Seismic data processing techniques of carbonate rocks in Tarim Basin. Lithologic Reservoirs. 2008. 20(4): 109-112 (in Chinese)

Williams M and Jenner E. Interpreting seismic data in the presence of azimuthal anisotropy or azimuthal anisotropy in the presence of seismic interpretation. The Leading Edge. 2002. 21(8): 771-774

Wu G H, Li Q M, Zhang B S, et al. Structural characteristics and exploration fields of No.1 faulted slope break in the Tazhong area. Acta Petrolei Sinica. 2005. 26(1): 27-30 (in Chinese)

Zhang G S, Ma G G, Song Y L, et al. Crack detection by utilizing full 3D P-wave data. Oil Geophysical Prospecting. 2004. 39(1): 41-44 (in Chinese)

Zhang Y. Application and prospects of the 3D seismic pre-stack time migration processing technique. Petroleum Exploration and Development. 2006. 33(5): 536-541 (in Chinese)

Zhao Z J, Wang Z M, Wu X N, et al. Genetic types and distribution forecast of available carbonate reservoirs in Ordovician in the central area of the Tarim Basin. Petroleum Geology and Experiment. 2007. 29(1): 40-46 (in Chinese)

Zhou J G, Deng H Y, Fan G Z, et al. Geological-geophysical model and prediction application of upper Ordovician Lianglitage reef-shoal reservoir in the Tazhong area, Tarim Basin. Marine Origin Petroleum Geology. 2008. 13(3): 17-23 (in Chinese)

(Edited by Hao Jie) 